\title{
Quantum light-matter interaction and controlled phonon scattering in a photonic Fano cavity
}

Denning, Emil V.; lles-Smith, Jake; Mork, Jesper

\section{Published in:}

Physical Review B

Link to article, DOI:

10.1103/PhysRevB.100.214306

Publication date:

2019

Document Version

Publisher's PDF, also known as Version of record

Link back to DTU Orbit

Citation (APA):

Denning, E. V., lles-Smith, J., \& Mork, J. (2019). Quantum light-matter interaction and controlled phonon scattering in a photonic Fano cavity. Physical Review B, 100(21), [214306].

https://doi.org/10.1103/PhysRevB.100.214306

\section{General rights}

Copyright and moral rights for the publications made accessible in the public portal are retained by the authors and/or other copyright owners and it is a condition of accessing publications that users recognise and abide by the legal requirements associated with these rights.

- Users may download and print one copy of any publication from the public portal for the purpose of private study or research.

- You may not further distribute the material or use it for any profit-making activity or commercial gain

- You may freely distribute the URL identifying the publication in the public portal 


\title{
Quantum light-matter interaction and controlled phonon scattering in a photonic Fano cavity
}

\author{
Emil V. Denning $\odot,{ }^{1}$ Jake Iles-Smith $\odot,{ }^{2}$ and Jesper Mork ${ }^{1, *}$ \\ ${ }^{1}$ Department of Photonics Engineering, DTU Fotonik, Technical University of Denmark, Building 343, 2800 Kongens Lyngby, Denmark \\ ${ }^{2}$ Department of Physics and Astronomy, University of Sheffield, Sheffield, S3 7RH, United Kingdom
}

(Received 31 July 2019; revised manuscript received 20 November 2019; published 10 December 2019)

\begin{abstract}
By using a Fano resonance as one of the mirrors in an optical cavity, a localized mode with a highly asymmetric line shape is obtained. Placing a single quantum emitter inside the cavity leads to a new regime of cavity quantum electrodynamics, where the light-matter interaction dynamics is fundamentally different from that observed in a conventional cavity with Lorenztian line shape. Furthermore, when the vibrational dynamics of the emitter is taken into account, an intricate phonon-photon interplay arises, and the optical interference induced by the Fano mirror significantly alters the leakage of energy into vibrational modes. We demonstrate that this control mechanism improves the maximum attainable indistinguishability of emitted photons, as compared to an equivalent cavity with a conventional mirror.
\end{abstract}

DOI: 10.1103/PhysRevB.100.214306

\section{INTRODUCTION}

The Fano effect arises from the interference between a continuum of modes and a localized resonance [1]. A photonic variant of the phenomenon can be realized by placing a nanocavity close to a waveguide with a partially transmitting element, as shown in Fig. 1(a). In this configuration, photons in the waveguide can propagate directly through the partially transmitting element or via the cavity. The resulting interference leads to a strong and asymmetric frequency dependence of the transmittivity through the system [2] [see Fig. 1(b)]. By replacing one of the mirrors in a conventional Fabry-Pérot cavity with such a Fano mirror, one may construct an exotic nanophotonic structure-a so-called Fano cavity. The interference between the two dissipation paths of the Fano mirror leads to a quasilocalized cavity mode with an asymmetric line shape that differs qualitatively from the Lorentzian lineshape of a conventional Fabry-Pérot cavity. Indeed, in the limit where the two paths exhibit perfect destructive interference, this mode becomes a true bound state in the continuum [3], which has recently been studied with a nonlinear atomic mirror that allows for excitation of the bound mode through a two-photon process [4,5]. Such Fano cavities are of growing interest in the field of integrated nanophotonics [6], where Fano lasers with unique dynamics $[7,8]$ and nonreciprocal elements [9] have been demonstrated. However, there are a number of open questions regarding how the non-Lorentzian nature of the cavity will impact the emission properties of a single quantum emitter.

In this paper, we extend the description of a Fano cavity into the regime of cavity quantum electrodynamics (cQED), where we consider a single solid-state emitter placed in the center of the cavity. This theory allows us to directly investigate the impact that the non-Lorentzian lineshape of the Fano cavity has on the optical properties of the emitter, and

\footnotetext{
*jesm@fotonik.dtu.dk
}

crucially demonstrate that the coupling between a solid-state emitter and its vibrational environment can be engineered by structuring the optical local density of states (LDOS). This effect is observed through drastic modifications to the phonon sideband present in solid-state emitters [10], which we show becomes highly sensitive to the shape of the LDOS of the nanophotonic structure, and is able to suppress phonon emission [see Fig. 1(c)], resulting in photon indistinguishabilities that surpass standard Lorentzian cavities.

\section{FANO CAVITY}

When studying light-matter coupling in CQED, quantisation of the cavity mode is of paramount importance. Therefore a central question in the analysis of the Fano cavity is how to fully represent the structured LDOS in regimes of potentially strong light-matter coupling. Recently, a general quantisation scheme based on symmetrized quasinormal modes has been presented, which relies on a numerical calculation of the electromagnetic modes of the system [11]. In contrast, we make use of a simple and intuitive approach that provides an analytical expression for the LDOS of the Fano cavity. We then develop a mapping that extracts the key features of the LDOS and incorporates them into an enlarged system Hamiltonian. This mapping generates a pair of dissipative bosonic modes [see Fig. 1(d)] which are coupled in order to capture the predominant interference pathways, and thus the non-Lorentzian behavior of the Fano cavity. Resolving such interference features requires at least two coupled modes, and thus cannot be analysed with any single-mode mapping technique such as the reaction coordinate master equations $[12,13]$. Our two-mode representation allows us to fully account for strong-coupling effects between the emitter and the Fano cavity, while simultaneously capturing the nonMarkovian dynamics arising from interactions with the vibrational phonon environment of the host lattice [14,15].

In the rotating wave approximation, the Hamiltonian describing the interaction between a single two-level emitter and 


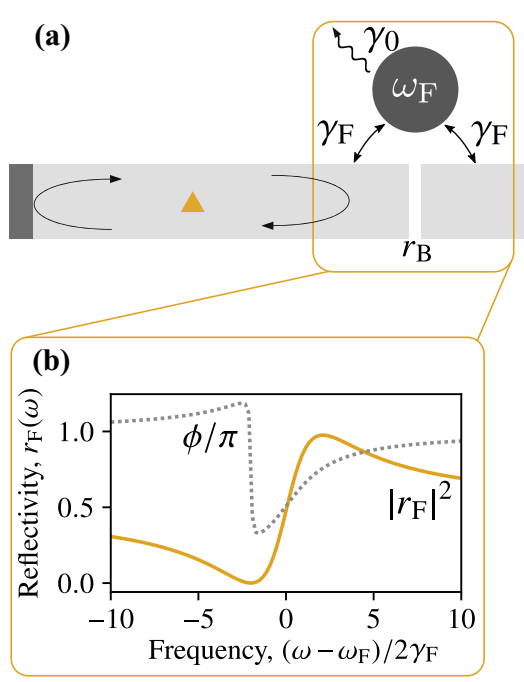

(c)

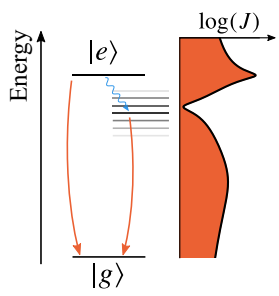

(d)

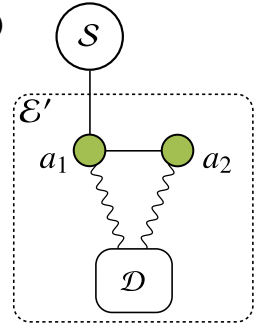

FIG. 1. (a) Fano cavity consisting of a waveguide with a fully reflecting mirror in the left end and a Fano mirror in the right end. (b) Squared modulus (orange solid) and phase (grey dotted) of the Fano mirror reflectivity, with $\gamma_{0}=0.05 \gamma_{\mathrm{F}}$ and $r_{\mathrm{B}}=-1 / \sqrt{2}$. (c) Level structure of quantum emitter $(|e\rangle$ and $|g\rangle)$ and phonon modes along with optical local density of states, $J$. (d) The photonic structure is mapped to a generic configuration, $\mathcal{E}^{\prime}$, consisting of two coupled discrete modes, dissipating into a common reservoir.

the electromagnetic field is $H_{\mathrm{EF}}=\sum_{\mu}\left(g_{\mu} a_{\mu}^{\dagger} \sigma+g_{\mu}^{*} a_{\mu} \sigma^{\dagger}\right)$, where $a_{\mu}$ is the bosonic annihilation operator for the $\mu^{\text {th }}$ mode of the field, $\sigma=|g\rangle\langle e|$ is the emitter transition operator between the excited $(|e\rangle)$ and ground $(|g\rangle)$ states, and $g_{\mu}$ is the coupling constant. The spectral density, $J(\omega)=$ $2 \pi \sum_{\mu}\left|g_{\mu}\right|^{2} \delta\left(\omega-\omega_{\mu}\right)$, with $\omega_{\mu}$ the frequency of the $\mu$ th mode, fully characterizes the interaction. This spectral density is equivalent to the LDOS of the electromagnetic field, up to a factor of $\pi d^{2} \omega /\left(\epsilon_{0} \hbar\right)$, where $d$ is the dipole moment of the emitter [16]. For optical frequencies, this factor is approximately constant over the relevant frequency range, and we shall in this paper generally refer to $J(\omega)$ as the local density of states. The LDOS can be calculated using an extension of Refs. [17-19] where the right mirror is a Fano mirror with reflectivity $r_{\mathrm{F}}(\omega)=r_{\mathrm{B}}+[-i(\omega-$ $\left.\left.\omega_{\mathrm{F}}\right) / 2 \gamma_{\mathrm{F}}+\gamma_{\mathrm{t}} / 2 \gamma_{\mathrm{F}}\right]^{-1}\left[-r_{\mathrm{B}}+i P t_{\mathrm{B}}\right][2,9,20]$ (see details in Appendix A). Here, $r_{\mathrm{B}}$ is the bare reflectivity of the partially transmitting element in the waveguide; $\gamma_{\mathrm{F}}$ is the coupling rate between the nanocavity and the waveguide, which is taken equal on both sides of the cavity; $\gamma_{0}$ is the intrinsic loss rate of the nanocavity; $\gamma_{\mathrm{t}}=2 \gamma_{\mathrm{F}}+\gamma_{0}$ is the total loss rate; $\omega_{\mathrm{F}}$ is the resonant frequency of the nanocavity and $P$ is the parity $( \pm 1)$ of the Fano mirror, determining whether the reflectivity minimum is on the blue- or red-detuned side of the maximum $[2,21]$. When the emitter is placed in the middle of the cavity, the spectral density becomes (see Appendix B)

$$
J(\omega)=\Gamma_{0} \operatorname{Re}\left[\frac{\left(1+r_{0} e^{i \omega / \Delta)}\right)\left(1+r_{\mathrm{F}}(\omega) e^{i \omega / \Delta}\right)}{1-r_{0} r_{\mathrm{F}}(\omega) e^{2 i \omega / \Delta}}\right],
$$

where $\Gamma_{0}$ is the spontaneous emission into the bare waveguide in the absence of mirrors, $r_{0}$ is the reflectivity of the conventional left mirror (here taken to be -1 ) and $\Delta / 2$ is the free (a)

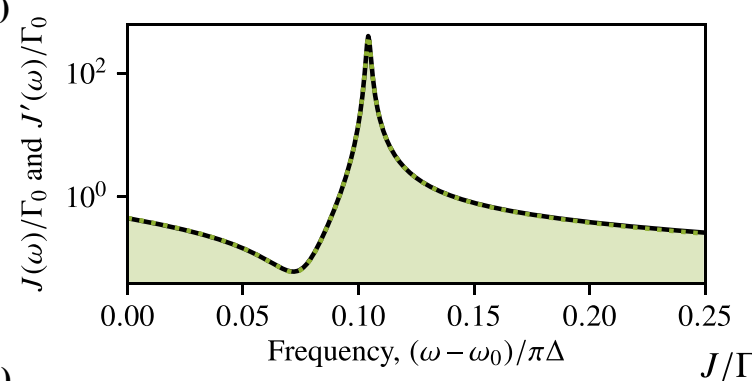

(b)

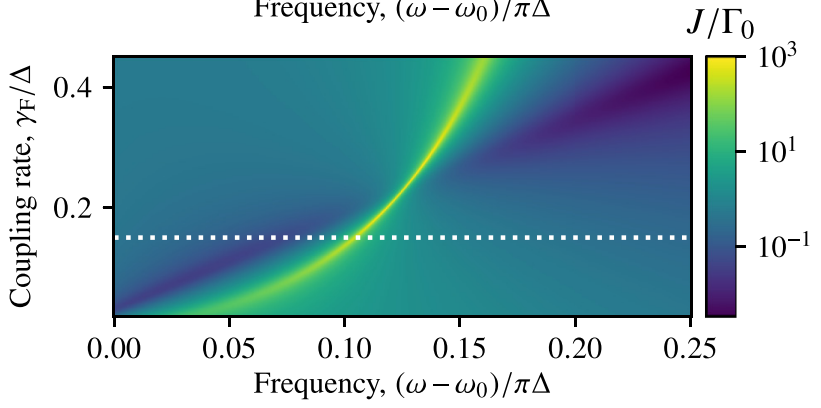

FIG. 2. (a) Frequency dependence of the local density of states, $J / \Gamma_{0}$, (black solid) corresponding to Eq. (1) and the spectral density, $J^{\prime} / \Gamma_{0}$, generated by the mapped structure (green dotted line, shaded area). Parameters: $r_{\mathrm{B}}=-1 / \sqrt{2}, P=+1, \gamma_{0}=10^{-3} \Delta, \omega_{0}=$ $101 \pi \Delta, \omega_{\mathrm{F}}=\omega_{0}-0.02 \pi \Delta$, and $\gamma_{\mathrm{F}}=0.15 \Delta$. (b) LDOS as a function of frequency and coupling rate. The white dotted line indicates the value of $\gamma_{\mathrm{F}}$ used in (a). The parameters are the same as in (a).

spectral range of the bare resonator set by $\Delta=c /(\bar{n} L)$, with $c$ the speed of light, $\bar{n}$ the effective waveguide group index and $L$ the cavity length. The interplay between the roundtrip phase in the cavity and the reflection phase of the Fano mirror gives rise to a resonance and an antiresonance appearing as a peak and a dip in the LDOS [see Fig. 2(a)]. Figure 2(b) shows how the LDOS depends on frequency and the coupling rate between the nanocavity and the waveguide. At the critical point where the antiresonance crosses over from the reddetuned to the blue-detuned side of the resonance, the peak corresponds to a bound state in the continuum [3]. At this point, the lifetime of this bound state is only limited by the intrinsic losses of the nanocavity.

\section{NON-MARKOVIAN FEW-MODE REPRESENTATION OF A FANO CAVITY}

Various techniques have been developed to perform the nontrivial task of constructing an accurate representation of a continuous bosonic environment in terms of a set of discrete modes. Reaction coordinate master equations have previously been used to extract a single damped bosonic mode from a continuous reservoir $[12,13]$. Such a representation, however, has so far only been successful in representing environments that are Lorentzian in nature, and therefore cannot capture the central features of the Fano cavity induced by interference. The pseudomode mapping associates a discrete mode with each complex pole of the spectral density [22-24]. This technique is exact but requires that all poles of the spectral density function are included in the mapping, because the sum of the residues must have a strictly vanishing real part. In the 
present paper, the spectral density has infinitely many poles, and therefore such an approach is infeasible. By including only a few poles in the relevant frequency range, the sum of the residues contains a nonzero real part, and as a result the pseudomode representation breaks down.

A more flexible approach allows representation of a bosonic environment as an infinite discrete chain of coupled harmonic oscillators [25], with the possibility of truncating the chain after a finite number of links [26]. In order to be efficient, the chain needs to represent the spectral density accurately with only a few links, or rely on sophisticated numerical techniques to calculate the dynamics of the reduced system $[27,28]$. In Appendix D, we describe how the electromagnetic environment can be mapped to a chain, and show the performance of this approach when describing the Fano cavity. We find that the accuracy of the mapping improves very slowly with the number of links included in the chain, and no satisfactory mapping was established for chain lengths up to 22. We attribute this poor convergence to the interference between the two optical decay paths in the physical structure that generates the central features of the spectral density. This interference is very difficult to reproduce in a one-dimensional structure, such as the chain representation.

On a more general note, it was recently shown that the reduced dynamics of a quantum system $\mathcal{S}$ with a bosonic environment $\mathcal{E}$ is invariant under a substitution of $\mathcal{E}$ by a different bosonic environment $\mathcal{E}^{\prime}$, provided that the spectral densities of $\mathcal{E}$ and $\mathcal{E}^{\prime}$ are equal [29]. In most practical applications, the correspondence between the spectral densities of $\mathcal{E}$ and $\mathcal{E}^{\prime}$ cannot be exact, but their difference within a relevant frequency range determined by $\mathcal{S}$ serves as a measure for equivalence of a given mapping. This strategy can be exemplified by the canonical representation of a QED emittercavity system by the dissipative Jaynes-Cummings model, where the cavity is described by a single mode with a finite lifetime.

To describe the asymmetric Fano cavity mode, in contrast, it is necessary to use two modes [see Fig. 1(d)]. Based on the physical structure of the Fano cavity, where two cavity modes (the Fabry-Pérot mode in the waveguide and the nanocavity) interfere in their common dissipation channel, we propose a quantum-optical representation with a similar structure. In this representation, the light-matter interaction of the emitter and cavity modes with annihilation operators $A=\left(a_{1}, a_{2}\right)^{T}$ is governed by the master equation

$$
\dot{\rho}=-i\left[H_{\mathrm{E}}+A^{\dagger} \Omega A+A^{\dagger} G \sigma+G^{\dagger} A \sigma^{\dagger}, \rho(t)\right]+\mathcal{D}\left(K^{\dagger} A\right) .
$$

Here, $H_{\mathrm{E}}=\omega_{e g} \sigma^{\dagger} \sigma$ is the free evolution Hamiltonian of the emitter, $\mathcal{D}(x)=x \rho(t) x^{\dagger}-\frac{1}{2}\left(x^{\dagger} x \rho(t)+\rho(t) x^{\dagger} x\right)$ is the Lindblad dissipator and

$$
\Omega=\left[\begin{array}{cc}
\omega_{1} & V \\
V^{*} & \omega_{2}
\end{array}\right], G=\left[\begin{array}{l}
g \\
0
\end{array}\right], K=\left[\begin{array}{c}
\sqrt{\kappa_{1}} \\
\sqrt{\kappa_{2}}
\end{array}\right] .
$$

We have introduced the emitter-cavity coupling strength $g$, the resonant frequency $\omega_{i}$ and leakage rate $\kappa_{i}$ for the $i$ th-cavity mode, and the intercavity coupling strength $V=V_{0} e^{i \theta}$. To accurately reproduce the correct LDOS of the Fano cavity, the interference between the two modes appearing in the dissipator is crucial [cf. Fig. 1(d)]. This is essentially due to the underlying interference induced by the Fano mirror that is responsible for the emergence of the cavity mode.

To assess the question of how well the two-mode representation reproduces the physical spectral density_or LDOSof the Fano cavity, we calculate the effective spectral density generated by the $A$-modes, $J^{\prime}(\omega)$. The parameters of the quantum-optical model are determined by minimising the error $\epsilon=\int_{W} d \omega\left[J(\omega)-J^{\prime}(\omega)\right]^{2}$, over a finite frequency window, $W$, around the emitter resonance. The spectral density of the discrete mode representation is calculated as $J^{\prime}(\omega)=$ $g^{2} \int_{-\infty}^{\infty} d \tau\left\langle a_{1}(\tau) a_{1}^{\dagger}\right\rangle e^{i \omega \tau}$, where $\langle\cdot\rangle$ denotes the free evolution of the cavity modes under Eq. (2) in the absence of the emitter. With analytic expressions for $J$ and $J^{\prime}$, we have access to the analytic continuation of the functions in the complex plane, which provides an efficient way to establish the mapping between the LDOS and the parameters of the few-mode representation $\left(\omega_{i}, \kappa_{i}, V, g\right)$. This requires that the positions of the complex poles of $J^{\prime}$ and $J$ coincide, and the imaginary parts of the residue sums are equal. These requirements determine five out of the seven parameters in the discrete-mode representation. The remaining two parameters are determined numerically by minimising $\epsilon$. The LDOS in Eq. (1) features an infinite number of discrete poles corresponding to the Fabry-Pérot resonances. However, the emitter is only sensitive to the LDOS in the vicinity of its transition frequency. For cavities with length scales of a few micrometers or shorter, the free spectral range is much larger than any features in the spectral response of the emitter and phonon modes, covering a few meV. As such, we find that features of the bound mode in the Fano cavity are very well described by two nearby poles of $J$, separated from the remaining poles by $\sim \pi \Delta$. In Fig. 2(a), we provide an example of how the mapped spectral density $J^{\prime}$ (green dashed line and shaded area) reproduces the LDOS $J$ (black solid line) with a high precision. A recent related work presents a similar technique based on a numerical search for the optimal parameters for a coupled network of harmonic oscillators to represent a given spectral density [30].

\section{ELECTRON-PHONON INTERACTIONS IN A FANO-CAVITY}

Interactions with longitudinal acoustic phonons are described by adding Hamiltonians representing the free energy of the phonons, $H_{\mathrm{P}}=\sum_{\mathbf{q}} v_{\mathbf{q}} b_{\mathbf{q}}^{\dagger} b_{\mathbf{q}}$, and the interaction energy with the emitter $H_{\mathrm{EP}}=\sigma^{\dagger} \sigma \sum_{\mathbf{q}} M_{\mathbf{q}}\left(b_{\mathbf{q}}^{\dagger}+b_{\mathbf{q}}\right)$ [31-33]. The phonon interactions are characterized by the spectral density $J_{\mathrm{P}}(v)=\sum_{\mathbf{q}} M_{\mathbf{q}}^{2} \delta\left(v-v_{\mathbf{q}}\right)=\alpha v^{3} \exp \left[-v^{2} / v_{c}^{2}\right]$, with overall coupling strength $\alpha$ and cutoff frequency $v_{c}[34,35]$. To account for the non-Markovian emitter-phonon correlations, we use the polaron transformation $[14,15,31,35,36]$ to derive a master equation (see Appendix E1). The resulting master equation is a modification of Eq. (2) in which the lightmatter coupling rate $g$ is effectively reduced and an additional dissipator is introduced. We furthermore add a dissipator accounting for spontaneous emission into the radiation modes $\Gamma_{\mathrm{R}} L(\sigma)[37,38]$.

The photon indistinguishability is an important measure of the coherence properties of the light emitted from a cQED system $[14,15,33,39,40]$. In the present situation, the 
indistinguishability reveals how strongly the photonic structure is able to modify the phonon dynamics by shaping the phonon sideband or enhancing/suppressing the zero phonon line. Here, the emission from the system is studied by choosing an initial state where the emitter is excited and the electromagnetic field is in the vacuum state, and then calculating the optical spectrum as the system relaxes and a single photon is emitted. Of particular interest is the two-color spectrum of light emitted from the system through the Fano mirror, $S\left(\omega, \omega^{\prime}\right)$ [15]. This two-color spectrum is essentially the Fourier transform of the two-time first order correlation function of the emitted light. It gives the observable emission spectrum, $\bar{S}(\omega)$, through the relation $\bar{S}(\omega)=S(\omega, \omega)$. Furthermore, it allows calculating the photon indistinguishability $[15,19]$

$$
\mathcal{I}=\left[2 P / \Gamma_{0}\right]^{-2} \int_{-\infty}^{\infty} d \omega \int_{-\infty}^{\infty} d \omega^{\prime}\left|S\left(\omega, \omega^{\prime}\right)\right|^{2},
$$

where $P=\left(\Gamma_{0} / 2\right) \int_{-\infty}^{\infty} d \omega \bar{S}(\omega)$ is the emitted energy into the detection channel. In a multiphoton experiment with $n$ subsequent two-photon interference events, the accumulated visibility scales as $\mathcal{I}^{n} \simeq 1-n \delta$, where $\delta:=1-\mathcal{I}$ is the distinguishibility, which quantifies the decoherence-induced error [41]. The two-color spectrum is related to the dipole spectrum of the emitter,

$$
S_{0}\left(\omega, \omega^{\prime}\right)=\int_{-\infty}^{\infty} d t \int_{-\infty}^{\infty} d t^{\prime} e^{i\left(\omega t-\omega^{\prime} t^{\prime}\right)}\left\langle\sigma^{\dagger}(t) \sigma\left(t^{\prime}\right)\right\rangle,
$$

through the optical Green's function as $S(\omega)=$ $G^{*}(\omega) G\left(\omega^{\prime}\right) S_{0}\left(\omega, \omega^{\prime}\right)$, where

$$
G(\omega)=\frac{\left[1+r_{0} e^{i \omega / \Delta}\right] t_{\mathrm{F}}(\omega)}{1-r_{0} r_{\mathrm{F}}(\omega) e^{2 i \omega / \Delta}}
$$

and $t_{\mathrm{F}}(\omega)$ is the transmittivity of the Fano mirror.

Figure 3(a) shows the photon indistinguishability as a function of nanocavity coupling rate and emitter transition frequency, corresponding to parameter variations over the LDOS shown in Fig. 2(b). The behavior of the indistinguishability over the parameter space shows a rich structure, which is mainly a result of the interplay between the electromagnetic LDOS and the phonon sideband. Along the dotted line, the emitter transition frequency is tuned to the resonance of the Fano cavity, meaning that emission is funnelled into the zerophonon line. In the lower part of the plot, the LDOS antiresonance is located on the red-detuned side of the resonance and thereby suppresses emission into the phonon sideband while enhancing emission into the zero phonon line. The combination of these effects leads to a minimal distinguishability of 0.010 (indicated with a white dot). The emission spectrum corresponding to these parameters is shown in Fig. 3(b) (solid orange; shading). To understand how this spectrum arises, the bulk emission spectrum is indicated (thin blue) along with the electromagnetic LDOS (grey dashed). This demonstrates how the antiresonance leads to a spectral hole in the phonon sideband.

In Appendix F, we show that the minimal attainable distinguishability for the same optical structure with a conventional mirror is $\delta=0.011[15,19]$, found when the mirror reflectivity is optimized to $r \simeq 0.99$. In this optimal regime, most of the
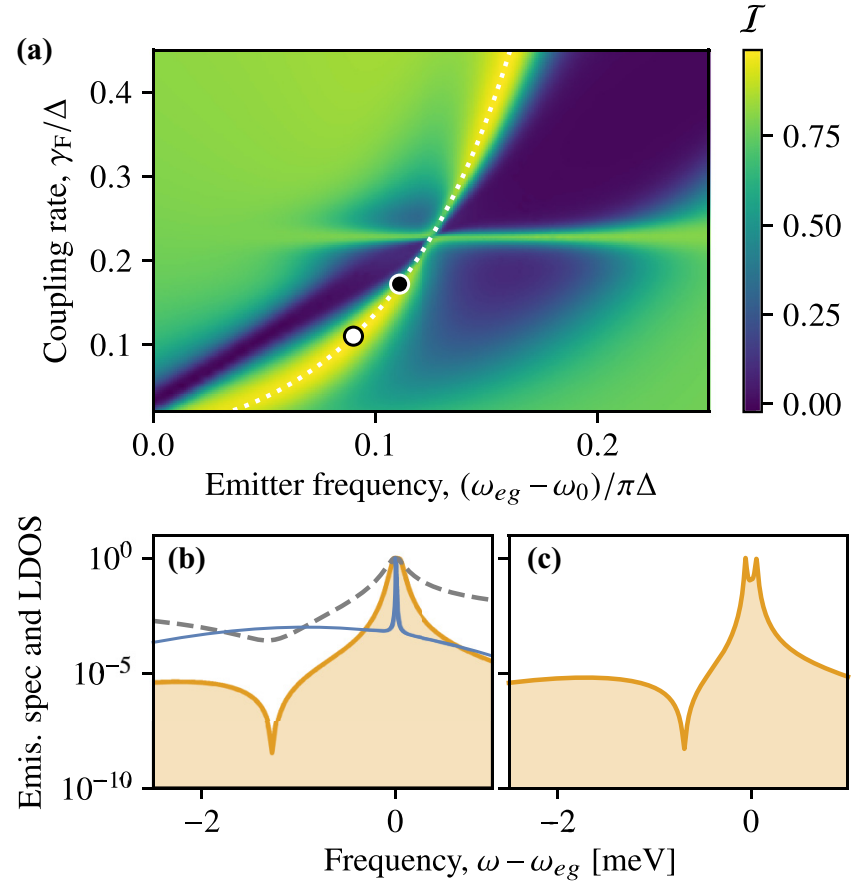

FIG. 3. (a) Photon indistinguishability (color scale) for varying emitter frequency, $\omega_{\text {eg }}$, and nanocavity coupling rate, $\gamma_{\mathrm{F}}$. The dotted white line traces the peak of the LDOS in Fig. 2 b. Parameters: $\Delta=10 \mathrm{meV}, \Gamma_{0}=0.6 \mu \mathrm{eV}, \Gamma_{\mathrm{R}}=0.03 \mu \mathrm{eV}, \alpha=$ $0.069 \mathrm{meV}^{-2}, v_{c}=1.45 \mathrm{meV}, T=4 \mathrm{~K}$ and otherwise as in Fig. 2. (b) Emission spectrum (solid orange line and shaded area) for optimal parameters with $\delta=0.010$ corresponding to the white dot in panel (a) along with the LDOS (grey dashed) and the bulk emission spectrum (blue solid line). All quantities are normalized to their peak value. (c) Emission spectrum for parameters corresponding to the black dot in panel (a).

emitted light is funnelled into the zero-phonon line, for both the Fano and Fabry-Pérot cavities. As a result, the spectral hole in the sideband present for the Fano cavity removes only a small fraction of the incoherent sideband emission, which is responsible for the reduction in distinguishability.

Figure 3(c) shows the emission spectrum for parameters corresponding to the black dot in Fig. 3(a), where the cavity lifetime is long enough for the system to enter the strongcoupling regime and two polariton peaks can be seen along with the spectral hole in the phonon sideband. In this regime, phonons reduce the indistinguishability by driving transitions between the polaritons $[15,33,39]$. If the emitter frequency is blue-shifted relative to the resonance line in Fig. 3(a), the LDOS peak will lie in the sideband, thereby increasing the phonon sideband emission, followed by a reduction of indistinguishability. Similarly, the low-indistinguishability area in Fig. 3(a) on the blue-detuned side of the resonant line arises because the emitter transition lies on the cavity antiresonance, thereby suppressing emission into the zero-phonon line.

\section{CONCLUSION}

In conclusion, we have analysed the quantum light-matter interactions between a single emitter and an optical Fano cavity. To this end, we have developed a mapping technique 
that allows to accurately represent the non-Lorentzian features of the cavity. We have demonstrated that coupling to the Fano cavity leads to rich and complex optical behavior, and in particular shown how the antiresonance of the Fano cavity can shape the phonon sideband, thereby increasing the photon indistinguishability. The suppression of vibrationally assisted photon emission enforced by the antiresonance in a Fano cavity is expected to be more pronounced in the case of a localized, spectrally sharp phonon resonance, as compared to the bulk, broadband phonon environment studied here. Such a situation may be encountered in emitters based on defect centers [42].

During the preparation of this manuscript, we became aware of related work [43] that presents a quantum optical description of the Fano mirror with applications in optomechanical cooling.

\section{ACKNOWLEDGMENTS}

E.V.D. and J.M. acknowledge funding from the Danish Council for Independent Research (Grant No. DFF-418100416), from Villum Fonden (Grant No. 8692), and from the European Research Council (ERC) under the European Union Horizon 2020 Research and Innovation Programme (Grant No. 834410 Fano). J.I.-S. acknowledges support from the Royal Commission for the Exhibition of 1851.

\section{APPENDIX A: REFLECTIVITY AND TRANSMITTIVITY OF FANO MIRROR}

Here, we calculate the reflectivity and transmittivity of the bare Fano mirror for a general situation, where the sidecoupled nanocavity couples to the semi-infinite left and right waveguide segments with different rates, $\gamma_{1}, \gamma_{2}$, and corresponding phases, $\theta_{1}, \theta_{2}$, (see Fig. 4). The reflectivity and transmittivity of the partially transmitting element are denoted by $r_{\mathrm{B}}$ and $t_{\mathrm{B}}$ and the intrinsic loss rate of the nanocavity is $\gamma_{0}$, e.g., representing vertical scattering losses.

To analyze this system, we use coupled mode theory as in Refs. [20,44-46], for the for the four in- and outgoing field amplitudes in the waveguide, $s_{i}$, as indicated in Fig. 4, and in the resonant mode of the nanocavity, $a$,

$$
\begin{aligned}
\frac{\mathrm{d} a}{\mathrm{~d} t} & =\left(-i \omega_{\mathrm{F}}-\gamma_{t}\right) a+\left(\begin{array}{ll}
d_{1} & d_{2}
\end{array}\right)\left(\begin{array}{c}
s_{1+} \\
s_{2+}
\end{array}\right), \\
\left(\begin{array}{l}
s_{1-} \\
s_{2-}
\end{array}\right) & =C\left(\begin{array}{l}
s_{1+} \\
s_{2+}
\end{array}\right)+\left(\begin{array}{l}
d_{1} \\
d_{2}
\end{array}\right) a, \quad C=\left(\begin{array}{cc}
r_{\mathrm{B}} & -i t_{\mathrm{B}} \\
-i t_{\mathrm{B}} & r_{\mathrm{B}}
\end{array}\right),
\end{aligned}
$$

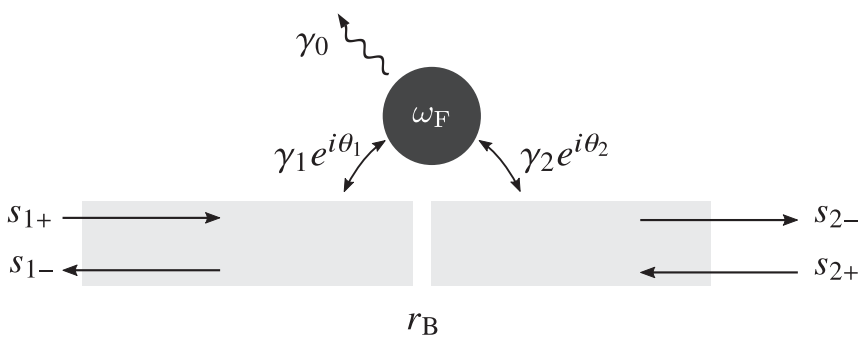

FIG. 4. Fano mirror with in- and outgoing field amplitudes $s_{i}$ indicated. with $\gamma_{t}=\sum_{i} \gamma_{i}, d_{i}=\sqrt{2 \gamma_{i}} e^{i \theta_{i}}$ and $\omega_{\mathrm{F}}$ is the resonance frequency of the cavity. Fourier transforming the equations, setting $s_{2+}=0$ and defining the amplitude reflectivity and transmittivity as $r_{\mathrm{F}}(\omega)=s_{1-} / s_{1+}, t_{\mathrm{F}}(\omega)=s_{2-} / s_{1+}$, we find

$$
\begin{aligned}
& r_{\mathrm{F}}(\omega)=r_{\mathrm{B}}+\frac{d_{1}^{2}}{-i\left(\omega-\omega_{\mathrm{F}}\right)+\gamma_{t}}, \\
& t_{\mathrm{F}}(\omega)=-i t_{\mathrm{B}}+\frac{d_{1} d_{2}}{-i\left(\omega-\omega_{\mathrm{F}}\right)+\gamma_{t}} .
\end{aligned}
$$

Using the relation $D=-C D^{*}[44]$, with

$$
D=\left(\begin{array}{ll}
d_{1} & 0 \\
d_{2} & 0
\end{array}\right) \text {, }
$$

we arrive at the expressions

$$
\begin{array}{r}
r_{\mathrm{F}}(\omega)=r_{\mathrm{B}}+2 \frac{i t_{\mathrm{B}} \sqrt{\gamma_{1} \gamma_{2}} e^{i\left(\theta_{1}-\theta_{2}\right)}-r_{\mathrm{B}} \gamma_{1}}{-i\left(\omega-\omega_{\mathrm{F}}\right)+\gamma_{t}}, \\
t_{\mathrm{F}}(\omega)=-i t_{\mathrm{B}}+2 \frac{i t_{\mathrm{B}} \gamma_{2}-r_{\mathrm{B}} \sqrt{\gamma_{1} \gamma_{2}} e^{i\left(\theta_{2}-\theta_{1}\right)}}{-i\left(\omega-\omega_{\mathrm{F}}\right)+\gamma_{t}} .
\end{array}
$$

The phases are not independent of the coupling parameters, but are given as [7,9]

$$
\begin{aligned}
\cos 2 \theta_{1} & =\frac{1}{2} \frac{t_{\mathrm{B}}^{2}}{r_{\mathrm{B}}}\left(\frac{\gamma_{2}}{\gamma_{1}}-1\right)-r_{\mathrm{B}}, \\
\sin 2 \theta_{1} & =P t_{\mathrm{B}} \frac{\sqrt{4 \gamma_{1} \gamma_{2}-t_{\mathrm{B}}^{2}\left(\gamma_{1}+\gamma_{2}\right)^{2}}}{2 \gamma_{1} r_{\mathrm{B}}}, \\
e^{i\left(\theta_{1}-\theta_{2}\right)} & =\sqrt{\gamma_{1} / \gamma_{2}} \frac{1}{i t_{\mathrm{B}}}\left(e^{2 i \theta_{1}}+r_{\mathrm{B}}\right)
\end{aligned}
$$

with $P$ the parity of the resonance, determined by the symmetry properties of the optical mode in the side-coupled cavity [21] and the position of the partially transmitting element relative to the nanocavity [6].

\section{Symmetric couplings}

If we now assume that the couplings are symmetric, $\gamma_{1}=$ $\gamma_{2}=: \gamma_{\mathrm{F}}$, we can reduce Eqs. (A2) and (A4) to

$$
\begin{aligned}
& r_{\mathrm{F}}(\omega)=r_{\mathrm{B}}+\frac{2 \gamma_{\mathrm{F}} e^{2 i \theta_{1}}}{-i\left(\omega-\omega_{\mathrm{F}}\right)+\gamma_{t}}, \\
& t_{\mathrm{F}}(\omega)=-i t_{\mathrm{B}}+\frac{2 \gamma_{\mathrm{F}}\left(i t_{\mathrm{B}}-r_{\mathrm{B}} e^{i\left(\theta_{2}-\theta_{1}\right)}\right)}{-i\left(\omega-\omega_{\mathrm{F}}\right)+\gamma_{t}} .
\end{aligned}
$$

These expressions can be further simplified by using the relations Eq. (A6), where we get $\cos 2 \theta_{1}=-r_{\mathrm{B}}, \sin 2 \theta_{1}=$ $P t_{\mathrm{B}}$, and $e^{i\left(\theta_{1}-\theta_{2}\right)}=P$. The reflectivity and transmittivity then become

$$
\begin{aligned}
& r_{\mathrm{F}}(\omega)=r_{\mathrm{B}}+\frac{-r_{\mathrm{B}}+i P t_{\mathrm{B}}}{-i\left(\omega-\omega_{\mathrm{F}}\right) / 2 \gamma_{\mathrm{F}}+\gamma_{t} / 2 \gamma_{\mathrm{F}}}, \\
& t_{\mathrm{F}}(\omega)=-i t_{\mathrm{B}}+\frac{i t_{\mathrm{B}}-P r_{\mathrm{B}}}{-i\left(\omega-\omega_{\mathrm{F}}\right) / 2 \gamma_{\mathrm{F}}+\gamma_{t} / 2 \gamma_{\mathrm{F}}} .
\end{aligned}
$$

\section{APPENDIX B: LOCAL DENSITY OF STATES OF ELECTROMAGNETIC FIELD IN FANO CAVITY}

Following Refs. [17,19], we consider a waveguide supporting a single guided transverse mode, with two mirrors 
(a)

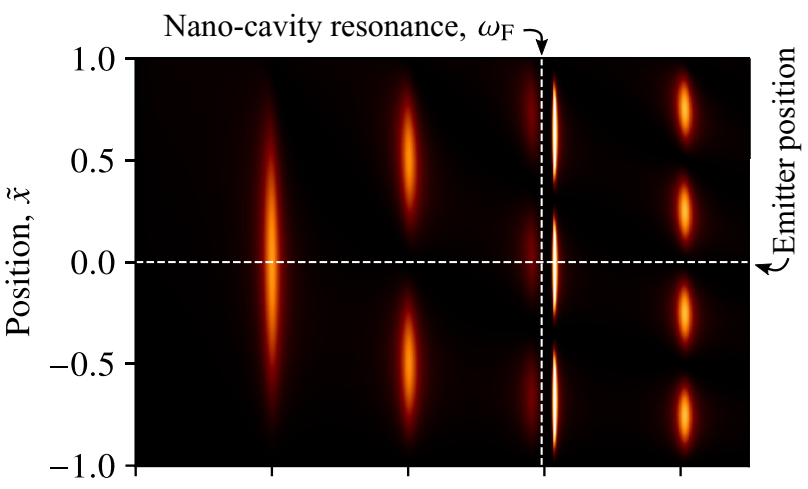

(b)

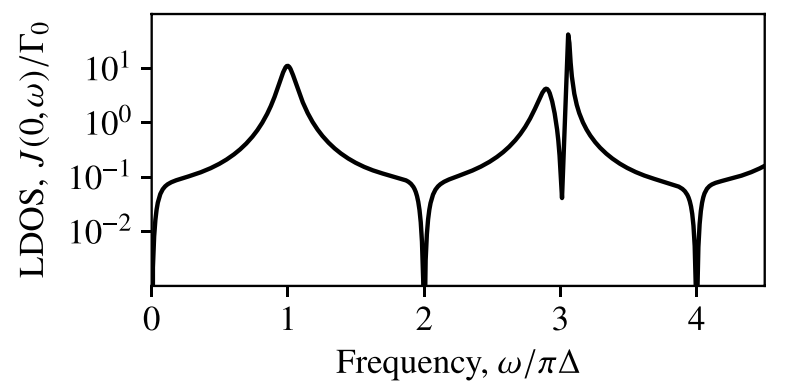

FIG. 5. (a) LDOS of the Fano cavity as a function of position, $\tilde{x}$, and frequency, $\omega$, normalized to the free spectral range, $\Delta$. The resonance frequency of the side-coupled nanocavity and the emitter position are indicated with vertical and horizontal dashed lines, respectively. (b) LDOS as a function of frequency taken at the position in the middle of the cavity region, $\tilde{x}=0$. The parameters for both panels are $r_{\mathrm{B}}=-1 / \sqrt{2}, \gamma_{1}=\gamma_{2}=0.05 \Delta, P=+1, \omega_{F}=$ $2.98 \Delta$, and $\gamma_{0}=10^{-3} \Delta$.

forming a cavity. In the region between the two mirrors, the local density of states (LDOS) of the electromagnetic field associated with the cavity mode is

$$
J(x, \omega)=\Gamma_{0} \operatorname{Re} \frac{\left[1+\tilde{r}_{1}(x, \omega)\right]\left[1+\tilde{r}_{2}(x, \omega)\right]}{1-\tilde{r}_{1}(x, \omega) \tilde{r}_{2}(x, \omega)},
$$

where $x$ is the longitudinal position ( $x= \pm L / 2$ corresponding to the position of the two mirrors, respectively), $\tilde{r}_{1}(x, \omega)$ and $\tilde{r}_{2}(x, \omega)$ are the complex reflectivities of the left and right mirror in the cavity, respectively. These are defined such that $\tilde{r}_{i}(x, \omega)$ also accounts for the propagation from the position of the emitter $x$ to the $i$ th mirror and back to $x$. Taking the left mirror (index 1) to be perfectly reflecting with a reflectance phase $\phi_{1}$ and the right mirror (index 2) to be a Fano resonance with reflectivity $r_{\mathrm{F}}(\omega)$,

$$
\begin{aligned}
& \tilde{r}_{1}(x, \omega)=\exp i\left[(1+\tilde{x}) \omega \bar{n} L / c+\phi_{1}\right], \\
& \tilde{r}_{2}(x, \omega)=r_{\mathrm{F}}(\omega) \exp i[(1-\tilde{x}) \omega \bar{n} L / c],
\end{aligned}
$$

where $c$ is the speed of light and $\tilde{x}=2 x / L$ is a dimensionless position along the waveguide axis, such that $\tilde{x}=-1$ corresponds to the position of the left mirror and $\tilde{x}=+1$ of the right mirror. The free spectral range of the resonator is then given by $\Delta / 2=c /(2 \bar{n} L)$ (such that the corresponding free spectral range for angular frequencies $\omega$ is $\pi \Delta$ ).

Figure 5(a) shows the LDOS of the Fano cavity $J(\tilde{x}, \omega)$ as a function of position and frequency. Far detuned from the resonance of the side-coupled cavity (indicated by a dashed vertical line) the partially transmitting element and the left mirror generate simple Fabry-Perot resonances with low quality factor separated by the free spectral range $\pi \Delta$. Around the resonance of the side-coupled cavity, Fano interference effects give rise to a sharp dip in the LDOS located close to a sharp peak. This is more clearly seen in Fig. 5(b), where the LDOS for the cavity midpoint $(\tilde{x}=0)$ is plotted.

\section{APPENDIX C: TWO-MODE MAPPING}

\section{Effective spectral density of few-mode reservoir}

The full Hamiltonian for the solid state emitter and the electromagnetic field is

$$
H=H_{\mathrm{E}}+\sum_{\mu} \omega_{\mu} a_{\mu}^{\dagger} a_{\mu}+\left[g_{\mu} a_{\mu}^{\dagger} \sigma+g_{\mu}^{*} a_{\mu} \sigma^{\dagger}\right],
$$

where $H_{\mathrm{E}}$ accounts for the free evolution of the emitter as well as the vibrational dynamics of the lattice. The mapped environment consists of two coupled cavity modes, described by bosonic annihilation operators $a_{1}$ and $a_{2}$, both coupled to a common auxiliary bosonic reservoir (labeled by the symbol $\xi)$ with annihilation operators $\alpha_{\mu}$. The emitter couples to the mapped environment only through the $a_{1}$ cavity mode (with the real coupling rate $g$ ). The full Hamiltonian for this system $H^{\prime}$ is

$$
\begin{aligned}
H^{\prime}= & H_{\mathrm{E}}+\sum_{i} \omega_{i} a_{i}^{\dagger} a_{i}+\left[g a_{1} \sigma^{\dagger}+V a_{1}^{\dagger} a_{2}+\text { H.c. }\right] \\
& +\left[q_{1} a_{1}^{\dagger}+q_{2} a_{2}^{\dagger}\right] \sum_{\mu} f_{\mu} \alpha_{\mu}+\left[q_{1}^{*} a_{1}+q_{2}^{*} a_{2}\right] \sum_{\mu} f_{\mu}^{*} \alpha_{\mu}^{\dagger} \\
& +\sum_{\mu} \epsilon_{\mu} \alpha_{\mu}^{\dagger} \alpha_{\mu},
\end{aligned}
$$

where $\epsilon_{\mu}$ and $f_{\mu}$ are the frequency and overall coupling rate to the $\mu$ th mode in the auxiliary reservoir, $q_{i}$ are unitless complex numbers specifying the relative strength and phase of the mode-reservoir couplings, $V$ is a complex coupling rate between the two cavity modes and $\omega_{1}, \omega_{2}$ are their frequencies.

The light-matter coupling terms in both $H$ and $H^{\prime}$ are of the form $\sigma c^{\dagger}+\sigma^{\dagger} c$, where for $H$ we have $c=\sum_{\mu} g_{\mu}^{*} a_{\mu}$ and for $H^{\prime}$ we have $c=g a_{1}$. If the initial state of the optical environment is taken as the vacuum, the only nonzero environmental correlation function is $\left\langle c(\tau) c^{\dagger}\right\rangle$, where the expectation value $\langle\cdot\rangle$ is taken with respect to the free evolution of the environment. Following Ref. [29], the equivalence between the emitter dynamics generated by $H$ and $H^{\prime}$ is quantified as the similarity between the two correlation functions

$$
\begin{aligned}
\Lambda(\tau) & =\left\langle\sum_{\mu \mu^{\prime}} g_{\mu}^{*} g_{\mu^{\prime}} a_{\mu}(\tau) a_{\mu^{\prime}}^{\dagger}\right\rangle=\sum_{\mu}\left|g_{\mu}\right|^{2}\left\langle a_{\mu}(\tau) a_{\mu}^{\dagger}\right\rangle, \\
\Lambda^{\prime}(\tau) & =g^{2}\left\langle a_{1}(\tau) a_{1}^{\dagger}\right\rangle .
\end{aligned}
$$

Equivalently, their corresponding spectral densities, $J(\omega)=$ $\int_{-\infty}^{\infty} d \tau \Lambda(\tau) e^{i \omega \tau}$ (and similarly for $J^{\prime}$ and $\Lambda^{\prime}$ ) can be used instead. The spectral density of the original system, $J$, can be calculated as the LDOS from Appendix B. For the mapped system, we can calculate $\Lambda^{\prime}(\tau)$ using $H^{\prime}$ and by tracing out the auxiliary reservoir. Imposing the Markov approximation on the auxiliary reservoir, the master equation corresponding 
to Eq. (C2) is [47]

$$
\begin{aligned}
\dot{\rho}(t)= & -i\left[H_{\mathrm{E}}+\sum_{i} \omega_{i} a_{i}^{\dagger} a_{i}+\left(g a_{1} \sigma^{\dagger}+V a_{1}^{\dagger} a_{2}+\text { H.c. }\right), \rho(t)\right] \\
& +\kappa \mathcal{D}\left[q_{1} a_{1}+q_{2} a_{2}\right],
\end{aligned}
$$

where $\mathcal{D}(x)=x \rho(t) x^{\dagger}-\frac{1}{2}\left(x^{\dagger} x \rho(t)+\rho(t) x^{\dagger} x\right)$ and $\kappa(\omega)=$ $2 \pi \sum_{\mu}\left|f_{\mu}\right|^{2} \delta\left(\omega-\epsilon_{\mu}\right)$ taken frequency-independent, consistent with the Markov approximation.

To calculate the environmental correlation function $\Lambda^{\prime}(\tau)$, we simply set $H_{\mathrm{E}}=0, g=0$. Since the initial state of the environment is taken as the vacuum state, the system only explores a subspace of the two-cavity Fock space $\left\{\left|n_{1}, n_{2}\right\rangle\right\}$ spanned by the basis $\{|0,0\rangle,|1,0\rangle,|0,1\rangle\}$. In this basis, the Liouvillian corresponding to the RHS of the master equation, Eq. (C4), can be represented by a $9 \times 9$ matrix, which we shall denote by $\mathcal{L}$. Since the interaction with the auxiliary environment is taken to be Markovian, we can calculate $\Lambda^{\prime}(\tau)$ using the quantum regression theorem [47], allowing for analytic calculation of the corresponding spectral density by matrix exponentiation,

$$
\begin{aligned}
J^{\prime}(\omega) & =2 g^{2} \operatorname{Re}\left\{\int_{0}^{\infty} d \tau e^{i \omega \tau} \operatorname{Tr}\left[a_{1} e^{\mathcal{L} \tau}\left(a_{1}^{\dagger}|0,0\rangle\langle 0,0|\right)\right]\right\} \\
& =2 g^{2} \operatorname{Re}\left[\frac{2 i\left(\omega-\omega_{2}\right)-\kappa\left|q_{2}\right|^{2}}{2\left(\omega-\omega_{2}\right)\left(\omega-\omega_{1}+\frac{1}{2} i \kappa\left|q_{1}\right|^{2}\right)+\left(-2 V+i q_{2} q_{1}^{*} \kappa\right) V^{*}+i \kappa\left[V q_{1} q_{2}^{*}+\left|q_{2}\right|^{2}\left(\omega-\omega_{1}\right)\right]}\right] \\
& =2 g^{2} \operatorname{Re}\left[\frac{2 i\left(\omega-\omega_{2}\right)-\kappa_{2}}{2\left(\omega-\omega_{2}\right)\left(\omega-\omega_{1}\right)+i \kappa_{1}\left(\omega-\omega_{2}\right)+i \kappa_{2}\left(\omega-\omega_{1}\right)+2 i V_{0} \sqrt{\kappa_{1} \kappa_{2}} \cos \varphi-2 V_{0}^{2}}\right],
\end{aligned}
$$

where, in the last equality, we have have defined

$$
\begin{aligned}
q_{1} & =:\left|q_{1}\right| e^{i \phi_{1}}, \quad q_{2}=:\left|q_{2}\right| e^{i \phi_{2}}, \\
\kappa_{1}: & =\kappa\left|q_{1}\right|^{2}, \quad \kappa_{2}:=\kappa\left|q_{2}\right|^{2}, \\
V & =: V_{0} e^{i \theta}, \quad \varphi:=\theta+\phi_{1}-\phi_{2} .
\end{aligned}
$$

In particular, we see that the spectral density does not depend on all of the complex phases individually, but only on the phase difference $\varphi$. We might thus conveniently set $\phi_{1}=$ $\phi_{2}=0$ and thus $\theta=\varphi$. In this case, the dissipator in the master equation, Eq. (C4), simplifies as $\kappa \mathcal{D}\left[q_{1} a_{1}+q_{2} a_{2}\right] \rightarrow$ $\mathcal{D}\left[\sqrt{\kappa_{1}} a_{1}+\sqrt{\kappa_{2}} a_{2}\right]$.

\section{a. Analytic structure}

The two poles of $J^{\prime}$, denoted by $z_{ \pm}^{\prime}$, are found as the roots of the denominator in (C5),

$$
\begin{aligned}
z_{ \pm}^{\prime}= & \frac{1}{2}\left[2 \Omega-\frac{i}{2}\left(\kappa_{1}+\kappa_{2}\right)\right] \pm \sqrt{D}, \\
D= & V_{0}^{2}-\left[\frac{\kappa_{1}+\kappa_{2}}{4}\right]^{2}+\frac{i \Delta}{4}\left(\kappa_{1}-\kappa_{2}\right)+\frac{\Delta^{2}}{4} \\
& -i \sqrt{\kappa_{1} \kappa_{2}} V_{0} \cos \varphi
\end{aligned}
$$

where $\Omega:=\frac{1}{2}\left(\omega_{1}+\omega_{2}\right)$ and $\Delta:=\omega_{2}-\omega_{1}$. This allows us to write $J^{\prime}$ as

$$
J^{\prime}(\omega)=2 g^{2} \operatorname{Re}\left\{\frac{i\left(\omega-\omega_{2}\right)-\frac{1}{2} \kappa_{2}}{\left(\omega-z_{+}^{\prime}\right)\left(\omega-z_{-}^{\prime}\right)}\right\} .
$$

From this form, we see that $z_{ \pm}^{\prime}$ are simple poles, and the corresponding residues, $R_{ \pm}^{\prime}$, are thus

$$
\begin{aligned}
R_{ \pm}^{\prime} & =\lim _{\omega \rightarrow z_{ \pm}^{\prime}}\left(\omega-z_{ \pm}^{\prime}\right) \mathcal{J}(\omega) \\
& =g^{2} \frac{i\left(z_{ \pm}^{\prime}-\omega_{2}\right)-\frac{1}{2} \kappa_{2}}{z_{ \pm}^{\prime}-z_{\mp}^{\prime}} .
\end{aligned}
$$

\section{Determining the parameters of the mapped environment}

Rather than optimising all of the seven parameters $\left(g, \kappa_{1}, \kappa_{2}, V_{0}, \theta, \omega_{1}, \omega_{2}\right)$ freely using a numerical fitting routine, we exploit our access to analytical properties of $J$ and $J^{\prime}$.

By calculating the analytical continuation of $J$ in the complex plane in the vicinity of the Fano cavity resonance, two poles can be found (see Fig. 6), which we shall denote by $z_{ \pm}$. By numerically integrating $J(z)$ along a closed contour $C_{ \pm}$, enclosing a single pole $z_{ \pm}$, the residue of this pole $R_{ \pm}$can be calculated using the residue theorem as

$$
R_{ \pm}=\frac{1}{2 \pi i} \oint_{C_{ \pm}} d z J(z) .
$$

Choosing the contour $C_{ \pm}$to be a circle with radius $Z_{0}, C_{ \pm}=$ $\left\{z: \phi \rightarrow Z_{0} e^{i \phi}+z_{ \pm} \mid \phi \in[0,2 \pi]\right\}$, the residue becomes

$$
\frac{Z_{0}}{2 \pi} \int_{0}^{2 \pi} d \phi J\left(Z_{0} e^{i \phi}+z_{ \pm}\right)
$$

We now require

$$
z_{+}^{\prime}=z_{+}, z_{-}^{\prime}=z_{-} .
$$

Adding these two equations yields

$$
\begin{gathered}
\Omega=\frac{1}{2} \operatorname{Re}\left[z_{+}+z_{-}\right], \\
\kappa_{1}+\kappa_{2}=-2 \operatorname{Im}\left[z_{+}+z_{-}\right] .
\end{gathered}
$$

Subtracting the two equations (C12) yields $2 \sqrt{D}=z_{+}-z_{-}$. Squaring and taking the real and imaginary parts of this equation leads to

$$
\begin{gathered}
V_{0}^{2}-\left(\frac{\kappa_{1}+\kappa_{2}}{4}\right)^{2}+\frac{\Delta^{2}}{4}=\frac{1}{4} \operatorname{Re}\left[\left(z_{+}-z_{-}\right)^{2}\right], \\
\frac{\Delta}{4}\left(\kappa_{1}-\kappa_{2}\right)-\sqrt{\kappa_{1} \kappa_{2}} V_{0} \cos \varphi=\frac{1}{4} \operatorname{Im}\left[\left(z_{+}-z_{-}\right)^{2}\right] .
\end{gathered}
$$




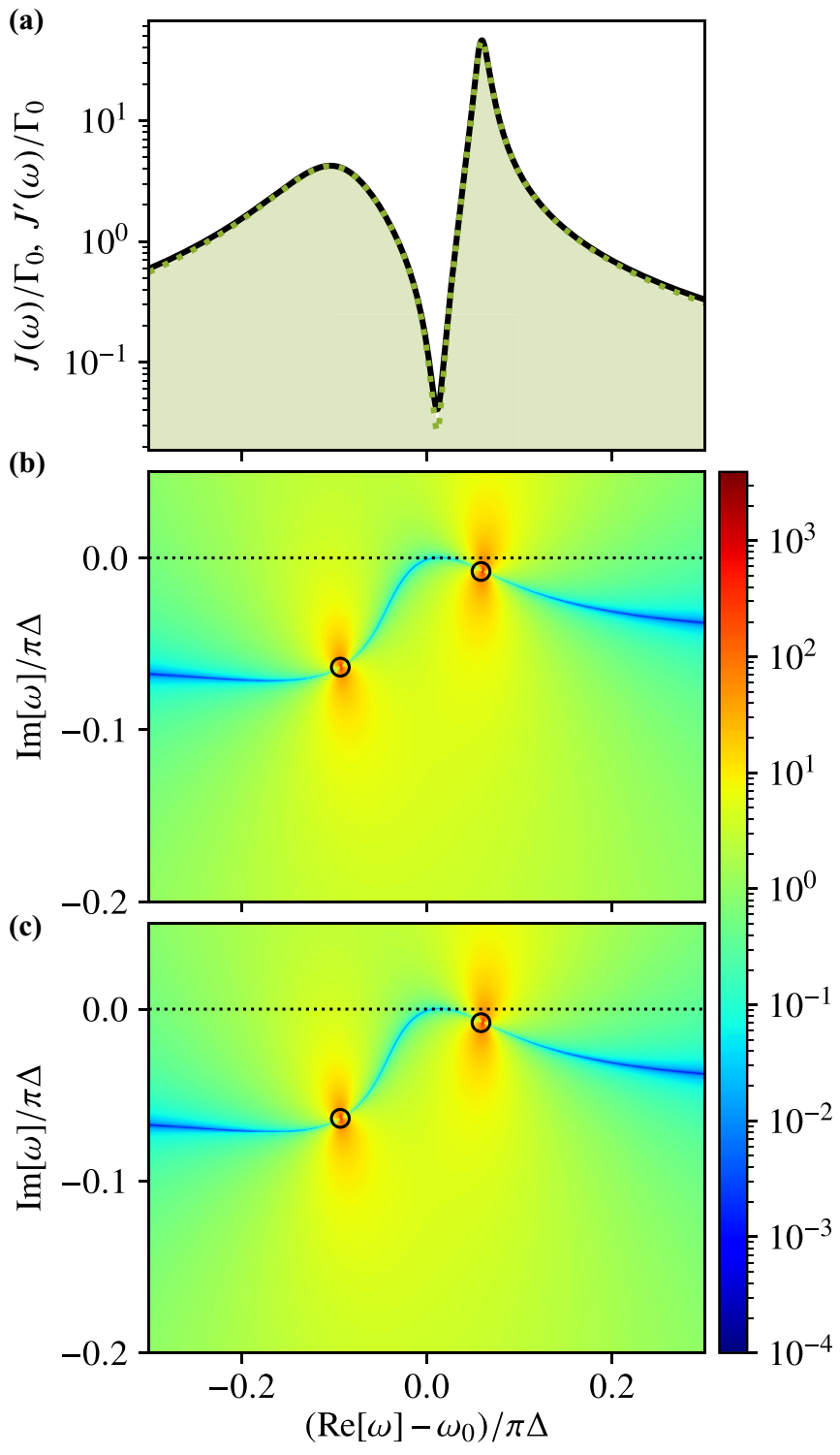

FIG. 6. (a) Original (black solid) and mapped (green dotted, shaded area) spectral densities, $J$ and $J^{\prime}$ as functions of real frequency, normalized to the bare spontaneous emission rate, $\Gamma_{0}$. [(b) and (c)] Analytic continuation of spectral densities, $|J| / \Gamma_{0}$ (b) and $\left|J^{\prime}\right| / \Gamma_{0}$ (c). The black circles indicate the poles. Parameters: $\gamma_{0}=10^{-} 3 \Delta, r_{\mathrm{B}}=-1 / \sqrt{2}, \omega_{0}=3 \Delta, \omega_{\mathrm{F}}=$ $\omega_{0}-0.02 \pi \Delta, P=1$, and $\gamma_{1}=\gamma_{2}=0.05 \Delta$.

Proceeding similarly with the residues $R_{ \pm}$and $R_{ \pm}^{\prime}$ would lead to four additional constraints, which would overdetermine the system. Alternatively, we use the restriction $\operatorname{Im}\left[R_{+}^{\prime}+R_{-}^{\prime}\right]=$ $\operatorname{Im}\left[R_{+}+R_{-}\right]$to generate the constraint

$$
g=\sqrt{\operatorname{Im}\left[R_{+}+R_{-}\right]} .
$$

Equations (C13)-(C17) gives five constraints, leaving two degrees of freedom. In our numerical implementation, we leave the two parameters $\Delta$ and $\kappa_{2}$ as free. We then choose the pair $\left(\Delta, \kappa_{2}\right)$ that minimizes error function $\epsilon=\int_{W} d \omega[J(\omega)-$ $\left.J^{\prime}(\omega)\right]^{2}$, where the integral runs over a frequency window $W$ that covers the central features of the LDOS in the vicinity of the resonance.

\section{APPENDIX D: CHAIN MAPPING}

Here we describe how the optical environment can be mapped to a discrete chain of coupled bosons with mode operators $a_{0}, a_{1}, \ldots, a_{n}$, such that the system is only coupled to the first mode $a_{0}$, as described in Refs. [25,26].

We first write the free field and emitter-field interaction Hamiltonians as a continuous integral over a frequency window $W$, which spans the important spectral features as

$$
\begin{gathered}
H_{\mathrm{F}}=\int_{W} d \omega \omega a^{\dagger}(\omega) a(\omega), \\
H_{\mathrm{EF}}=\int_{W} d \omega\left[h(\omega) a(\omega) \sigma^{\dagger}+\text { H.c. }\right],
\end{gathered}
$$

where the spectral density is related to the coupling strength as $|h(\omega)|^{2}=J(\omega) / 2 \pi$. According to Ref. [26], there exists a class of unitary transformations $U_{m}$ such that the transformed Hamiltonians are

$$
\begin{aligned}
\tilde{H}_{\mathrm{F}}= & \sum_{i=0}^{m-1} \Omega_{i} a_{i}^{\dagger} a_{i}+\sum_{i=0}^{m-2} q_{i+1}\left(a_{i}^{\dagger} a_{i+1}+a_{i+1}^{\dagger} a_{i}\right) \\
& +\int_{W} d \omega\left\{\omega \alpha^{\dagger}(\omega) \alpha(\omega)+\tilde{h}(\omega)\left[a_{m-1}^{\dagger} \alpha(\omega)+\text { H.c. }\right]\right\} \\
\tilde{H}_{\mathrm{EF}}= & q_{0}\left(\sigma a_{0}^{\dagger}+\sigma^{\dagger} a_{0}\right),
\end{aligned}
$$

where $a_{i}$ is a discrete set of $m$ bosonic modes coupled in a one-dimensional chain through the coefficients $q_{i}$. The first link of the chain $(i=0)$ is coupled to the emitter dipole, and the last link $(i=m-1)$ is coupled to a residual bosonic reservoir with spectral density $\tilde{J}(\omega)=2 \pi|\tilde{h}(\omega)|^{2}$ and annihilation operators $\alpha(\omega)$. References [25,26] describe in detail how to obtain the parameters $\Omega_{i}, q_{i}$ and the coupling function $\tilde{h}(\omega)$ from the spectral density $J(\omega)$. Ideally, the non-Markovian features of the electromagnetic environment should now be contained in the chain modes, and the residual reservoir should describe only Markovian dissipation effects. Roughly speaking, the complexity of the original spectral density $J(\omega)$ determines how many chain modes, $m$, are needed before the residual environment can be considered Markovian.

To calculate the dynamics of the emitter, we can derive a master equation for the reduced dynamics of the emitter and $m$ chain modes, tracing out the residual reservoir. First, it is convenient to split $\tilde{H}_{\mathrm{F}}$ into a part that describes the free evolution of the chain, $\tilde{H}_{\mathrm{F}}^{c}=\sum_{i=0}^{m-1} \Omega_{i} a_{i}^{\dagger} a_{i}+\sum_{i=0}^{m-2} q_{i+1}\left(a_{i}^{\dagger} a_{i+1}+\right.$ $\left.a_{i+1}^{\dagger} a_{i}\right)$, a part that describes the free evolution of the residual environment, $\tilde{H}_{\mathrm{F}}^{r}=\int_{W} d \omega \omega \alpha^{\dagger}(\omega) \alpha(\omega)$ and a part that couples the two, $\tilde{H}_{\mathrm{F}}^{I}=\int_{W} d \omega \tilde{h}(\omega)\left[a_{m-1}^{\dagger} \alpha(\omega)+\right.$ H.c. $]$. The Hamiltonian of the emitter and chain in the absence of the residual environment is then $\mathcal{H}:=H_{\mathrm{E}}+\tilde{H}_{\mathrm{EF}}+\tilde{H}_{\mathrm{F}}^{c}$.

We can then derive a Born-Markov master equation for the reduced density operator of the emitter and $m$-mode boson chain, under the secular approximation [47], and assuming that the residual reservoir is initially in the vacuum state. The secular approximation is needed to ensure positivity of the dynamics of the reduced density operator. Without enforcing secularity, we find unphysical behavior, such as negative effective spectral densities. The form of the secularized master 


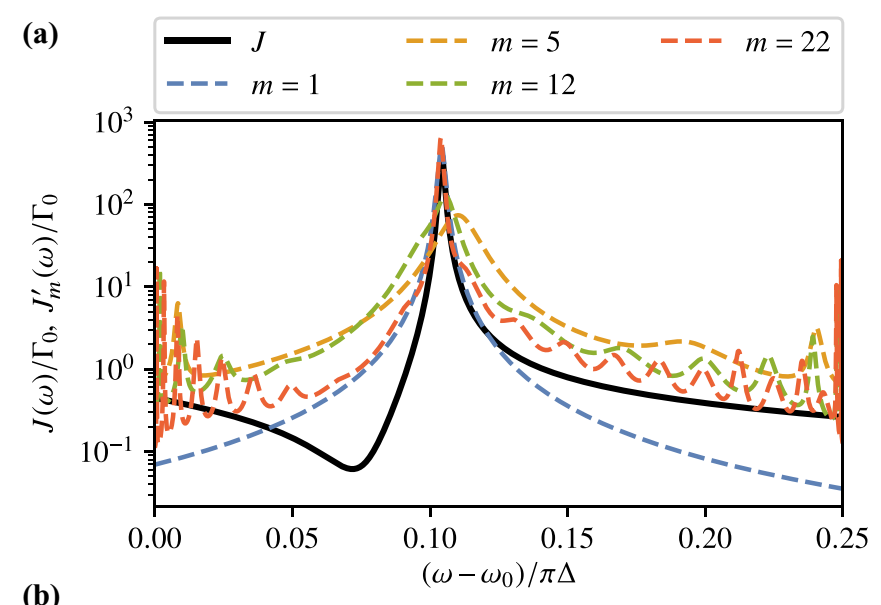

(b)

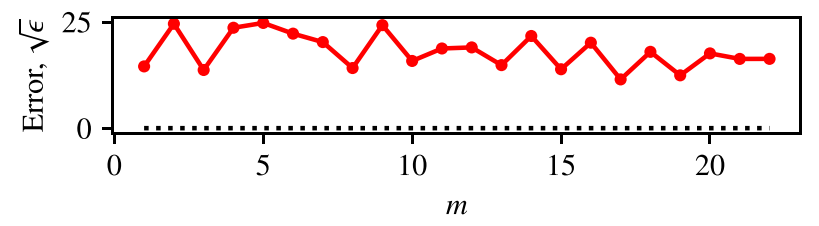

FIG. 7. (a) Physical spectral density, $J(\omega)$ (black solid), and effective spectral density generated by chain mapping, $J_{m}^{\prime}(\omega)$ (dashed) for chain lengths, $m$, up to 22. (b) Root mean square error between $J(\omega)$ and $J_{m}^{\prime}(\omega)$ as a function of $m$. The parameters for the Fano LDOS are the same as in Fig. 2(a). For comparison, the error of the two-mode mapping developed in the present paper is $\sqrt{\epsilon}=0.052$, shown with the black dotted line.

equation is

$$
\begin{aligned}
\dot{\rho}= & -i[\mathcal{H}, \rho] \\
& +\sum_{\nu} \gamma(v)\left(\tilde{a}_{m-1}^{v} \rho\left(\tilde{a}_{m-1}^{v}\right)^{\dagger}-\frac{1}{2}\left\{\left(\tilde{a}_{m-1}^{v}\right)^{\dagger} \tilde{a}_{m-1}^{v}, \rho\right\}\right) \\
& -i \Delta(v)\left[\left(\tilde{a}_{m-1}^{v}\right)^{\dagger} \tilde{a}_{m-1}^{v}, \rho\right],
\end{aligned}
$$

where $\tilde{a}_{m-1}^{v}$ is defined as $\tilde{a}_{m-1}^{v}=\sum_{E^{\prime}-E=v} \Pi(E) a_{m-1} \Pi\left(E^{\prime}\right)$ with $\Pi(E)$ the projection operator onto the eigenspace of $\mathcal{H}$ with eigenenergy $E$. The frequency-dependent dissipation rate $\gamma(v)$ and frequency renormalisation $\Delta(v)$ are given by

$$
\begin{gathered}
\gamma(v)=2 \pi \tilde{h}(v)^{2}, \\
\Delta(v)=\text { P.V. } \int_{W} d v^{\prime} \frac{\tilde{h}\left(v^{\prime}\right)^{2}}{v-v^{\prime}} .
\end{gathered}
$$

To assess the accuracy of the $m$-link chain representation of the electromagnetic environment, we can calculate the effective spectral density, $J_{m}^{\prime}(\omega)$, generated by the master equation, Eq. (D4). This is done, as described in Sec. C, by letting $\mathcal{H} \rightarrow H_{\mathrm{F}}^{c}$ in Eq. (D4) and calculating the resulting correlation function $\left\langle a_{0}(\tau) a_{0}^{\dagger}\right\rangle_{m}$. The Fourier transform of this correlation function is the spectral density, $J_{m}^{\prime}(\omega)=$ $q_{0}^{2} \int_{-\infty}^{\infty} d \tau\left\langle a_{0}(\tau) a_{0}^{\dagger}\right\rangle_{m} e^{i \omega \tau}$. Such a comparison is shown in Fig. 7(a), for chain lengths, $m$, up to 22. For $m=1$, the mapping produces a single Lorentzian peak at the position of Fano cavity resonance frequency, but does not capture the asymmetric line shape. For increasing $m$, the mapping slowly starts to describe the asymmetry of the resonance line, but at the same time produces significant parasitic oscillations in the spectral density.

\section{APPENDIX E: POLARON MASTER EQUATION AND DYNAMICS}

\section{Master equation}

The full Hamiltonian of the mapped optical environment and phonon reservoir is $H^{\prime}+H_{\mathrm{P}}+H_{\mathrm{EP}}$, where $H^{\prime}$ is defined in Eq. (C2), $H_{\mathrm{E}}=\omega_{e g} \sigma^{\dagger} \sigma$ describes the free evolution of the emitter, $H_{\mathrm{P}}=\sum_{\mathbf{q}} v_{\mathbf{q}} b_{\mathbf{q}}^{\dagger} b_{\mathbf{q}}$ describes the free evolution of the phonons and $H_{\mathrm{EP}}=\sigma^{\dagger} \sigma \sum_{\mathbf{q}} M_{\mathbf{q}}\left(b_{\mathbf{q}}+b_{\mathbf{q}}^{\dagger}\right)$ describes the emitter-phonon coupling. In the polaron frame, described by the unitary transformation $U_{\mathrm{P}}=|g\rangle\langle g|+| e\rangle\langle e| B_{+}$, with $B_{ \pm}=\exp \left[ \pm \sum_{\mathbf{q}} v_{\mathbf{q}}^{-1} M_{\mathbf{q}}\left(b_{\mathbf{q}}^{\dagger}-b_{\mathbf{q}}\right)\right]$, the Hamiltonian is $\hat{H}^{\prime}:=$ $U_{\mathrm{P}} H^{\prime} U_{\mathrm{P}}^{\dagger}=: \hat{H}_{0}+\hat{H}_{\mathrm{R}}+\hat{H}_{\mathrm{I}}$, where

$$
\hat{H}_{0}=\hat{\omega}_{e g} \sigma^{\dagger} \sigma+\sum_{i} \omega_{i} a_{i}^{\dagger} a_{i}+\hat{g} X+\left(V a_{1}^{\dagger} a_{2}+V^{*} a_{2}^{\dagger} a_{1}\right)
$$

describes the internal dynamics of the two-level system and the two mapped optical modes,

$$
\hat{H}_{\mathrm{R}}=\sum_{\mu} \epsilon_{\mu} \alpha_{\mu}^{\dagger} \alpha_{\mu}+\sum_{\mathbf{q}} v_{\mathbf{q}} b_{\mathbf{q}}^{\dagger} b_{\mathbf{q}}
$$

describes the free evolution of the total reservoir comprising the auxiliary electromagnetic environment and the phonon environment, and

$$
\begin{aligned}
\hat{H}_{\mathrm{I}}= & {\left[q_{1} a_{1}^{\dagger}+q_{2} a_{2}^{\dagger}\right] \sum_{\mu} f_{\mu} \alpha_{\mu}+\left[q_{1}^{*} a_{1}+q_{2}^{*} a_{2}\right] \sum_{\mu} f_{\mu}^{*} \alpha_{\mu}^{\dagger} } \\
& +g\left(X B_{X}+Y B_{Y}\right)
\end{aligned}
$$

describes the system-reservoir interactions. The quantities entering these expressions are defined as $X=\sigma^{\dagger} a_{1}+$ $\sigma a_{1}^{\dagger}, Y=i\left(\sigma^{\dagger} a_{1}-\sigma a_{1}^{\dagger}\right), B_{X}=\left(B_{+}+B_{-}-2 B_{0}\right) / 2, B_{Y}=$ $i\left(B_{+}-B_{-}\right) / 2, \hat{g}=B_{0} g$, where $B_{0}=\operatorname{Tr}\left[\rho_{\mathrm{P}}^{0} B_{+}\right]$is the expectation value of $B_{+}$with respect to the phonon thermal state, $\rho_{\mathrm{P}}^{0}=\exp \left[-\frac{1}{k_{\mathrm{B}} T} \sum_{\mathbf{q}} v_{\mathbf{q}} b_{\mathbf{q}}^{\dagger} b_{\mathbf{q}}\right] / \operatorname{Tr}\left\{\exp \left[-\frac{1}{k_{\mathrm{B}} T} \sum_{\mathbf{q}} v_{\mathbf{q}} b_{\mathbf{q}}^{\dagger} b_{\mathbf{q}}\right]\right\}$, with $k_{\mathrm{B}}$ and $T$ the Boltzmann constant and temperature, respectively. Tracing out the phonon environment and auxiliary electromagnetic environment leads to the second-order Born-Markov master equation for the reduced density operator $\rho$ [47]:

$$
\begin{aligned}
\dot{\rho}(t)= & -i\left[\hat{H}_{0}, \rho(t)\right] \\
& -\int_{0}^{\infty} d \tau \operatorname{Tr}_{\mathrm{R}}\left[H_{\mathrm{I}},\left[H_{\mathrm{I}}(-\tau), \rho(t) \otimes \rho_{\mathrm{P}}^{0} \otimes \rho_{\xi}^{0}\right]\right],
\end{aligned}
$$

where the reference state of the auxiliary electromagnetic environment is taken as the vacuum, $\rho_{\xi}^{0}=\bigotimes_{\mu}\left|0_{\mu}\right\rangle\left\langle 0_{\mu}\right|$, assuming temperatures significantly below the typical optical frequencies around the emitter resonance, $k_{\mathrm{B}} T \ll \omega_{e g}$. Writing this master equation out explicitly yields

$$
\dot{\rho}(t)=-i\left[\hat{H}_{0}, \rho(t)\right]+\mathcal{D}\left[\sqrt{\kappa_{1}} a_{1}+\sqrt{\kappa_{2}} a_{2}\right]+\mathcal{W},
$$

where the phonon-induced term is

$$
\mathcal{W}=g^{2}\left(\left[X, \rho(t) \Theta_{X}^{\dagger}\right]+\left[Y, \rho(t) \Theta_{Y}^{\dagger}\right]+\text { H.c. }\right)
$$

where $\Theta_{\zeta}=\int_{0}^{\infty} d \tau \zeta(-\tau) \Lambda_{\zeta}(\tau)$, with $\zeta=X, Y$ and $\zeta(-\tau)$ denoting the free Heisenberg picture time evolution, $\zeta(-\tau)=$ 
$e^{-i \hat{H}_{0} \tau} \zeta e^{+i \hat{H}_{0} \tau}$. The phonon correlation functions are $\Lambda_{X}(\tau)=$ $\frac{1}{2} B^{2}\left[e^{\phi(t)}+e^{-\phi(\tau)}-2\right], \Lambda_{Y}(\tau)=\frac{1}{2} B^{2}\left[e^{\phi(\tau)}-e^{-\phi(\tau)}\right] \quad$ with $\phi(\tau)=\int_{0}^{\infty} d v v^{-2} J_{\mathrm{P}}(v)[\operatorname{coth}(\beta \nu / 2) \cos v \tau-i \sin v \tau]$ and $\beta=1 /\left(k_{\mathrm{B}} T\right)$. The phonon spectral density is $J_{\mathrm{P}}(v)=\sum_{\mathbf{q}} M_{\mathbf{q}}^{2} \delta\left(v-v_{\mathbf{q}}\right)=\alpha v^{3} \exp \left[-v^{2} / \nu_{c}^{2}\right]$, with overall coupling strength $\alpha$ and cutoff frequency $v_{c}$.

\section{Dipole spectrum}

When calculating dynamics in the polaron frame, quantities of interest must be transformed back to the laboratory frame. In particular, the dipole correlation function, $\left\langle\sigma^{\dagger}(t) \sigma\left(t^{\prime}\right)\right\rangle_{\mathrm{LF}}$ (with subscript LF signifying expectation values in the laboratory frame) is calculated from the polaron frame (subscript PF) dynamics as [48]

$$
\left\langle\sigma^{\dagger}(t) \sigma\left(t^{\prime}\right)\right\rangle_{\mathrm{LF}}=\left\langle\sigma^{\dagger}(t) B_{+}(t) B_{-}\left(t^{\prime}\right) \sigma\left(t^{\prime}\right)\right\rangle_{\mathrm{PF}} .
$$

Assuming that the two-level system and phonons are weakly coupled in the polaron frame, this is approximated as

$$
\left\langle\sigma^{\dagger}(t) \sigma\left(t^{\prime}\right)\right\rangle_{\mathrm{LF}} \simeq\left\langle B_{+}(t) B_{-}\left(t^{\prime}\right)_{\mathrm{PF}}\left\langle\sigma^{\dagger}(t) \sigma\left(t^{\prime}\right)\right\rangle_{\mathrm{PF}},\right.
$$

and $\left\langle B_{+}(t) B_{-}\left(t^{\prime}\right)\right\rangle_{\mathrm{PF}}$ is approximated as the equilibrium phonon correlation function, $\left\langle B_{+}(t) B_{-}\left(t^{\prime}\right)\right\rangle_{\mathrm{PF}} \simeq B_{0}^{2} e^{\phi\left(t-t^{\prime}\right)}$.

The two-color emission spectrum $S\left(\omega, \omega^{\prime}\right)$, observed in the waveguide region on the right-hand side of the Fano mirror is related to the dipole correlation function as $[14,15]$

$$
S\left(\omega, \omega^{\prime}\right)=G(\omega) G\left(\omega^{\prime}\right) S_{0}\left(\omega, \omega^{\prime}\right)
$$

where $S_{0}\left(\omega, \omega^{\prime}\right)=\int_{-\infty}^{\infty} d t d t^{\prime}\left\langle\sigma^{\dagger}(t) \sigma\left(t^{\prime}\right)\right\rangle_{\mathrm{LF}} e^{i\left(\omega t-\omega^{\prime} t^{\prime}\right)}$ is the dipole spectrum and

$$
G(\omega)=\frac{\left(1+r_{0} e^{i \omega / \Delta}\right) t_{\mathrm{F}}(\omega)}{1-r_{0} r_{\mathrm{F}}(\omega) e^{2 i \omega / \Delta}}
$$

is the Green's function that connects the dipole spectrum with the spectrum of the electromagnetic field in the waveguide, transmitted through the Fano mirror [19].

\section{APPENDIX F: EQUIVALENT FABRY-PEROT CAVITY}

When both mirrors in the cavity are regular broadband mirrors, a simpler analysis can be carried out. The LDOS in

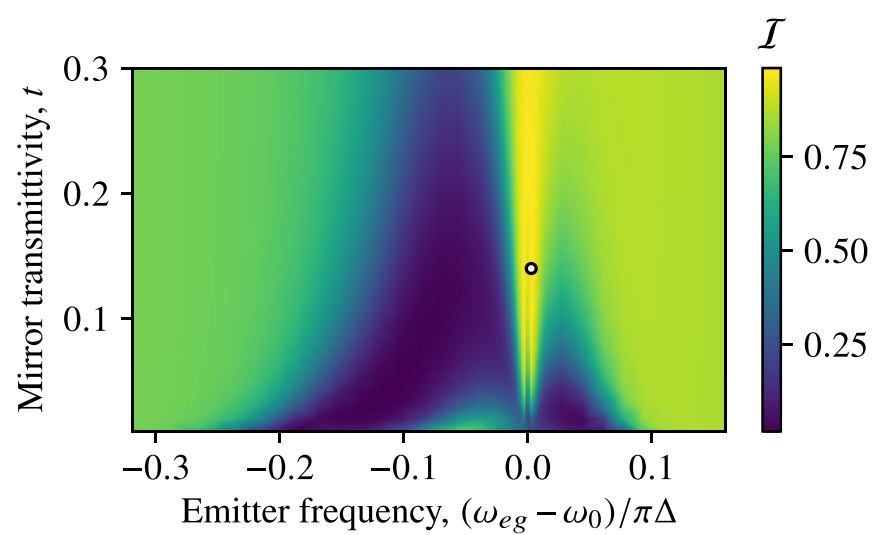

FIG. 8. Indistinguishability of Fabry-Perot cavity as a function of emitter frequency and right mirror transmittivity. Parameters: $\Delta=10 \mathrm{meV}, \Gamma_{0}=0.6 \mu \mathrm{eV}, \Gamma_{\mathrm{R}}=0.03 \mu \mathrm{eV}, \alpha=$ $0.069 \mathrm{meV}^{-2}, v_{c}=1.45 \mathrm{meV}$, and $T=4 \mathrm{~K}$. The white dot indicates the minimal distinguishability, $\delta=0.011$.

the middle position of the cavity is then

$$
J(\omega)=\Gamma_{0} \operatorname{Re}\left[\frac{\left(1+r_{0} e^{i \omega / \Delta}\right)\left(1+r e^{i \omega / \Delta}\right)}{1-r_{0} r e^{2 i \omega / \Delta}}\right],
$$

where $r$ is the reflectivity of the right mirror. Following the strategy from Ref. [19], a single cavity mode can be extracted from the LDOS with reflectivity-dependent emitter coupling strength, $g$, and decay rate, $\kappa$. The master equation describing the dynamics (including interactions with phonons) is then

$$
\dot{\rho}(t)=-i\left[B_{0} g\left(a \sigma^{\dagger}+a^{\dagger} \sigma\right), \rho(t)\right]+\kappa L(a)+\mathcal{W},
$$

where $a$ is the annihilation operator of the cavity mode and $\mathcal{W}$ is as in Eq. (E6), but with $X=a \sigma^{\dagger}+a^{\dagger} \sigma, Y=i\left(a \sigma^{\dagger}-\right.$ $\left.a^{\dagger} \sigma\right)$. The emission spectrum and indistinguishability is calculated using the same technique as for the Fano cavity, but with the Fabry-Pérot Green's function

$$
G(\omega)=\frac{\left(1+r_{2} e^{i \omega / \Delta}\right) t}{1-r_{0} r e^{2 i \omega / \Delta}}
$$

where $t=\sqrt{1-r^{2}}$ is the transmittivity of the right mirror.

Figure 8 shows the indistinguishability for a Fabry-Pérot cavity as a function of emitter frequency and transmittivity of the right mirror. Except from the right mirror, all the properties of the optical structure are the same as in Fig. 3 of the main text. The minimal distinguishability of 0.011 is indicated with a dot.
[1] U. Fano, Phys. Rev. 124, 1866 (1961).

[2] M. Heuck, P. T. Kristensen, Y. Elesin, and J. Mørk, Opt. Lett. 38, 2466 (2013).

[3] H. Friedrich and D. Wintgen, Phys. Rev. A 32, 3231 (1985).

[4] M. Cotrufo and A. Alù, Optica 6, 799 (2019).

[5] G. Calajó, Y.-L. L. Fang, H. U. Baranger, and F. Ciccarello, Phys. Rev. Lett. 122, 073601 (2019).
[6] D. Bekele, Y. Yu, K. Yvind, and J. Mork, Laser Photon. Rev. 1900054 (2019).

[7] Y. Yu, W. Xue, E. Semenova, K. Yvind, and J. Mork, Nat Photon. 11, 81 (2017).

[8] J. Mork, Y. Chen, and M. Heuck, Phys. Rev. Lett. 113, 163901 (2014).

[9] Y. Yu, Y. Chen, H. Hu, W. Xue, K. Yvind, and J. Mork, Laser Photon. Rev. 9, 241 (2015). 
[10] L. Besombes, K. Kheng, L. Marsal, and H. Mariette, Phys. Rev. B 63, 155307 (2001).

[11] S. Franke, S. Hughes, M. K. Dezfouli, P. T. Kristensen, K. Busch, A. Knorr, and M. Richter, Phys. Rev. Lett. 122, 213901 (2019).

[12] J. Iles-Smith, N. Lambert, and A. Nazir, Phys. Rev. A 90, 032114 (2014).

[13] J. Iles-Smith, A. G. Dijkstra, N. Lambert, and A. Nazir, J. Chem. Phys. 144, 044110 (2016).

[14] K. Roy-Choudhury and S. Hughes, Phys. Rev. B 92, 205406 (2015).

[15] J. Iles-Smith, D. P. S. McCutcheon, A. Nazir, and J. Mørk, Nat. Photon. 11, 521 (2017).

[16] P. Lodahl, S. Mahmoodian, and S. Stobbe, Rev. Mod. Phys. 87, 347 (2015).

[17] N. Gregersen, D. P. S. McCutcheon, J. Mørk, J.-M. Gérard, and J. Claudon, Opt. Express 24, 20904 (2016).

[18] M. V. Rybin, S. F. Mingaleev, M. F. Limonov, and Y. S. Kivshar, Sci. Rep. 6, 20599 (2016).

[19] E. V. Denning, J. Iles-Smith, A. D. Osterkryger, N. Gregersen, and J. Mork, Phys. Rev. B 98, 121306(R) (2018).

[20] J. W. Yoon and R. Magnusson, Opt. Express 21, 17751 (2013).

[21] A. D. Osterkryger, J. R. de Lasson, M. Heuck, Y. Yu, J. Mørk, and N. Gregersen, Opt. Lett. 41, 2065 (2016).

[22] B. M. Garraway, Phys. Rev. A 55, 2290 (1997).

[23] B. J. Dalton, S. M. Barnett, and B. M. Garraway, Phys. Rev. A 64, 053813 (2001).

[24] L. Mazzola, S. Maniscalco, J. Piilo, K.-A. Suominen, and B. M. Garraway, Phys. Rev. A 80, 012104 (2009).

[25] A. W. Chin, Á. Rivas, S. F. Huelga, and M. B. Plenio, J. Math. Phys. 51, 092109 (2010).

[26] M. P. Woods, R. Groux, A. W. Chin, S. F. Huelga, and M. B. Plenio, J. Math. Phys. 55, 032101 (2014).

[27] J. Prior, A. W. Chin, S. F. Huelga, and M. B. Plenio, Phys. Rev. Lett. 105, 050404 (2010).

[28] R. Rosenbach, J. Cerrillo, S. F. Huelga, J. Cao, and M. B. Plenio, New J. Phys. 18, 023035 (2016).
[29] D. Tamascelli, A. Smirne, S. F. Huelga, and M. B. Plenio, Phys. Rev. Lett. 120, 030402 (2018).

[30] F. Mascherpa, A. Smirne, A. D. Somoza, P. Fernández-Acebal, S. Donadi, D. Tamascelli, S. F. Huelga, and M. B. Plenio, arXiv:1904.04822.

[31] I. Wilson-Rae and A. Imamoğlu, Phys. Rev. B 65, 235311 (2002).

[32] G. D. Mahan, Many-Particle Physics (Springer Science \& Business Media, 2013).

[33] P. Kaer and J. Mørk, Phys. Rev. B 90, 035312 (2014).

[34] A. Nazir, Phys. Rev. B 78, 153309 (2008).

[35] A. Nazir and D. P. S. McCutcheon, J. Phys.: Condens. Matter. 28, 103002 (2016).

[36] D. P. S. McCutcheon and A. Nazir, New J. Phys. 12, 113042 (2010).

[37] D. P. S. McCutcheon and A. Nazir, Phys. Rev. Lett. 110, 217401 (2013).

[38] K. Roy-Choudhury and S. Hughes, Optica 2, 434 (2015).

[39] P. Kaer, T. R. Nielsen, P. Lodahl, A.-P. Jauho, and J. Mørk, Phys. Rev. Lett. 104, 157401 (2010).

[40] G. Hornecker, A. Auffèves, and T. Grange, Phys. Rev. B 95, 035404 (2017).

[41] A. Kiraz, M. Atatüre, and A. Imamoğlu, Phys. Rev. A 69, 032305 (2004).

[42] A. Alkauskas, B. B. Buckley, D. D. Awschalom, and C. G. Van de Walle, New J. Phys. 16, 073026 (2014).

[43] O. Černotík, A. Dantan, and C. Genes, Phys. Rev. Lett. 122, 243601 (2019).

[44] W. Suh, Z. Wang, and S. Fan, IEEE J. Quantum Electron. 40, 1511 (2004).

[45] K. X. Wang, Z. Yu, S. Sandhu, and S. Fan, Opt. Lett. 38, 100 (2013).

[46] P. T. Kristensen, J. R. de Lasson, M. Heuck, N. Gregersen, and J. Mørk, J. Lightwave Technol. 35, 4247 (2017).

[47] H.-P. Breuer and F. Petruccione, The Theory of Open Quantum Systems (Oxford University Press on Demand, 2002).

[48] C. Roy and S. Hughes, Phys. Rev. B 85, 115309 (2012). 